\title{
Onset and progression of diabetes in kidney transplant patients receiving everolimus or cyclosporine therapy: an analysis of two randomized, multicenter trials
}

Claudia Sommerer $^{1 *}$, Oliver Witzke ${ }^{2}$, Frank Lehner ${ }^{3}$, Wolfgang Arns ${ }^{4}$, Petra Reinke ${ }^{5}$, Ute Eisenberger ${ }^{6}$, Bruno Vogt $^{6}$, Katharina Heller ${ }^{7}$, Johannes Jacobi ${ }^{7}$, Markus Guba ${ }^{8}$, Rolf Stahl ${ }^{9}$, Ingeborg A. Hauser ${ }^{10}$, Volker Kliem ${ }^{11}$,

Rudolf P. Wüthrich ${ }^{12}$, Anja Mühlfeld ${ }^{13}$, Barbara Suwelack ${ }^{14}$, Michael Duerr ${ }^{15}$, Eva-Maria Paulus ${ }^{16}$, Martin Zeier ${ }^{1}$, Martina Porstner ${ }^{16 t}$ and Klemens Budde ${ }^{15+}$ on behalf of the ZEUS and HERAKLES study investigators

\begin{abstract}
Background: Conversion from calcineurin inhibitor (CNI) therapy to a mammalian target of rapamycin (mTOR) inhibitor following kidney transplantation may help to preserve graft function. Data are sparse, however, concerning the impact of conversion on posttransplant diabetes mellitus (PTDM) or the progression of pre-existing diabetes.

Methods: PTDM and other diabetes-related parameters were assessed post hoc in two large open-label multicenter trials. Kidney transplant recipients were randomized (i) at month 4.5 to switch to everolimus or remain on a standard cyclosporine (CSA)-based regimen (ZEUS, $n=300$ ), or (ii) at month 3 to switch to everolimus, remain on standard CNI therapy or convert to everolimus with reduced-exposure CSA (HERAKLES, $n=497$ ).

Results: There were no significant differences in the incidence of PTDM between treatment groups (log rank $p=0.97$ [ZEUS], $p=0.90$ [HERAKLES]). The mean change in random blood glucose from randomization to month 12 was also similar between treatment groups in both trials for patients with or without PTDM, and with or without pre-existing diabetes. The change in eGFR from randomization to month 12 showed a benefit for everolimus versus comparator groups in all subpopulations, but only reached significance in larger subgroups (no PTDM or no pre-existing diabetes).
\end{abstract}

Conclusions: Within the restrictions of this post hoc analysis, including non-standardized diagnostic criteria and limited glycemia laboratory parameters, these data do not indicate any difference in the incidence or severity of PTDM with early conversion from a CsA-based regimen to everolimus, or in the progression of pre-existing diabetes.

Trial registration: clinicaltrials.gov, NCT00154310 (registered September 2005) and NCT00514514 (registered August 2007); EudraCT (2006-007021-32 and 2004-004346-40).

Keywords: Diabetes, Everolimus, Kidney transplantation, TOR inhibitor, PTDM, Post-transplant

\footnotetext{
* Correspondence: claudia.sommerer@med.uni-heidelberg.de

+Martina Porstner and Klemens Budde contributed equally to this work.

${ }^{1}$ Department of Nephrology, University of Heidelberg, Im Neuenheimer Feld

162, 69120 Heidelberg, Germany

Full list of author information is available at the end of the article
}

(c) The Author(s). 2018 Open Access This article is distributed under the terms of the Creative Commons Attribution 4.0 International License (http://creativecommons.org/licenses/by/4.0/), which permits unrestricted use, distribution, and reproduction in any medium, provided you give appropriate credit to the original author(s) and the source, provide a link to the Creative Commons license, and indicate if changes were made. The Creative Commons Public Domain Dedication waiver (http://creativecommons.org/publicdomain/zero/1.0/) applies to the data made available in this article, unless otherwise stated. 


\section{Background}

Diabetic nephropathy is now the most frequent indication for kidney transplantation, accounting for approximately a third of kidney transplants in the US [1], and is set to grow in frequency as the prevalence of diabetes continues to grow [2, 3]. Additionally, under conventional immunosuppressive regimens, up to $20 \%$ of kidney transplant recipients develop posttransplant diabetes mellitus (PTDM) [4-6]. Both pre-existing diabetes [7, 8] and PTDM [9] are associated with an increased risk of cardiovascular events $[9,10]$ and inferior long-term survival, as well as morbidity from diabetes-related complications [7, 11]. The diabetogenic effect of calcineurin inhibitors (CNIs), particularly tacrolimus [4, 12] and steroids [5], can be compounded by maintenance steroid therapy, especially pulsed steroid therapy for the treatment of rejection [13]. In this unfavorable context, novel immunosuppressive regimens must be carefully evaluated in terms of their diabetogenic potential.

The mammalian target of rapamycin (mTOR) inhibitor agents sirolimus and everolimus have been widely assessed within a variety of regimens for de novo or delayed initiation following kidney transplantation [14]. mTOR inhibitors offer the potential for CNI sparing, which might be expected to lower the risk for PTDM [15]. However, results from the early era of sirolimus therapy in kidney transplantation raised concerns that the class may have an inherent diabetogenic effect $[16,17]$. In a large randomized trial of sirolimus published in 2006, Vitko et al. reported an increased rate of PTDM in patients randomized to a loading dose $(6 \mathrm{mg})$ and a fixed dose of $2 \mathrm{mg} /$ day versus mycophenolate mofetil when both were administered in combination with standarddose tacrolimus and steroids [18]. Although smaller trials using fixed sirolimus dosing $[19,20]$ or high sirolimus exposure targets [21] did not demonstrate any effect, an analysis of United States Renal Data System data from over 20,000 patients undergoing kidney transplantation during 1995-2003 concluded that patients treated with sirolimus were at increased risk of PTDM whether administered in combination with a $\mathrm{CNI}$ or an antimetabolite [22]. In contrast, a large meta-analysis published in 2006 found that use of mTOR inhibitors was not associated with any increased risk of developing insulin-treated PTDM compared to antimetabolite therapy [23].

As experience with mTOR inhibitors has grown, fixed dosing has been replaced by progressively lower trough concentration targets, and concomitant CNI exposure has been reduced [14]. Loading doses for sirolimus have typically become smaller, and no loading dose is required for everolimus due to its shorter half-life. In large, randomized trials undertaken recently, no increase in the rate of PTDM was observed in the sirolimus [24, 25] or everolimus [26, 27] treatment arms.
Conversion to an mTOR inhibitor from CNI therapy after the first 3-12 months post-transplant is an appealing immunosuppressive strategy, harnessing the potent immunosuppressive effect of CNIs during the period of highest risk for rejection but taking advantage of the reduced nephrotoxicity associated with mTOR inhibitors [28]. To date, no analyses are available concerning the impact of conversion to an mTOR inhibitor on PTDM or the progression of pre-existing diabetes. We report here a post hoc analysis of diabetic parameters in two large, multicenter trials (ZEUS [29] and HERAKLES [30]) in which de novo kidney transplant recipients were randomized to either convert to everolimus in a CNI-free regimen or to remain on a standard cyclosporine (CsA)-based regimen, or in one study to alternatively switch to everolimus with reduced-exposure CsA.

\section{Methods}

\section{Study design and conduct}

This was a post hoc analysis of data from two 12-month, prospective, open-label, multicenter, randomized trials of de novo kidney transplant recipients (ZEUS [29] and HERAKLES [30]).The objective of the analysis was to compare the incidence and severity of PTDM, and progression of pre-existing diabetes, to month 12 post-transplant in patients receiving everolimus-based CNI-free maintenance immunosuppression versus those who continued to receive a standard CsA-based regimen or everolimus with reduced-exposure CsA. In both studies, patients received standard-exposure CsA with enteric-coated mycophenolate sodium (EC-MPS) and steroids from time of transplant, and were randomized to continue the CsA-based regimen or convert to everolimus at 4.5 months (ZEUS) [29] or 3 months (HERAKLES) [30] post-transplant. In one of the studies (HERAKLES), there was a third treatment arm in which patients received reduced-exposure CsA with everolimus targeting a lower exposure range.

\section{Patients}

The inclusion and exclusion criteria in the two studies were identical other than a lower maximum age for recipients and donors in the ZEUS study (65 years) versus the HERAKLES study (70 years). The minimum recipient and donor ages were 18 and 5 years, respectively, in both studies. Key exclusion criteria at time of study entry were more than one previous kidney transplant, loss of a previous graft due to immunological reasons, multiorgan transplantation, donation after cardiac death, and previous or current panel reactive antibodies $>25 \%$. At the time of randomization, additional exclusion criteria were graft loss, severe (Banff grade $\geq$ III), recurrent or steroid-resistant rejection prior to randomization, proteinuria $>1 \mathrm{~g} /$ day and dialysis dependency. In both studies, 
patients with uncontrolled diabetes mellitus that in the opinion of the investigator would interfere with the appropriate conduct of the study were excluded. A full list of inclusion and exclusion criteria is shown in Additional file 1: Table S1.

\section{Immunosuppression}

All patients received induction with basiliximab (Simulect ${ }^{\circ}$, Novartis Pharma, Nürnberg, Germany). CsA (Sandimmun Optoral $^{\circ}$, Novartis Pharma, Germany) was administered to all patients, with a target trough concentration in both studies of $150-220 \mathrm{ng} / \mathrm{mL}$ from the time of transplant to randomization. All patients received EC-MPS $1440 \mathrm{mg} /$ day (myfortic ${ }^{\ominus}$, Novartis Pharma, Germany), and steroids administered according to local practice from the time of transplant to month 12 .

For patients in the standard-CsA arms, CsA target trough concentrations after randomization were 120$180 \mathrm{ng} / \mathrm{mL}$ to month 6 , and $100-150 \mathrm{ng} / \mathrm{mL}$ thereafter; EC-MPS was continued to month 12 . In the CNI-free everolimus arms of both studies, everolimus was initiated at a dose of $1.5 \mathrm{mg}$ then adjusted to target a trough concentration of 5-10 ng/mL, and EC-MPS was continued to month 12. In the ZEUS study, conversion from CsA to everolimus took place stepwise over a period of up to four weeks starting at month 4.5. In the HERAKLES study, conversion took place at month 3 and was completed within $24 \mathrm{~h}$. In the patients randomized to everolimus with reduced-exposure CsA in the HERAKLES study, EC-MPS was discontinued and everolimus was started on the day of randomization with CsA dose unchanged, then on the following day CsA dose was adjusted to target $50-75 \mathrm{ng} / \mathrm{mL}$ thereafter. In this group, the everolimus target range was $3-8 \mathrm{ng} / \mathrm{mL}$.

\section{Evaluation}

This post hoc analysis compared the following outcomes between treatment groups up to month 12 post-transplant within the ZEUS and HERAKLES trials: the incidence of PTDM; requirement for hypoglycemic therapy (insulin or non-insulin); change in random blood glucose; estimated GFR (eGFR) at month 12 and the change in eGFR from randomization to month 12 . Other than eGFR at month 12 , none of these endpoints were pre-specified in the study protocols. Data were analyzed according to whether patients did or did not have pre-existing diabetes and subsequently did or did not develop PTDM. PTDM was defined as diabetes reported by the investigator as an adverse event at any point after transplantation in a patient not categorized as diabetic at baseline (i.e. at time of transplant). Patients were categorized as diabetic if diabetes was listed in the medical history by the investigator at the time of study entry. There were no pre-specified laboratory criteria for PTDM or pre-existing diabetes. Data on the use of insulin or other antidiabetic therapies were obtained via standard reporting procedures for concomitant medication at each study visit. If treatment with such drugs was started, investigators were required to document any adverse events, as per Good Clinical Practice guidelines. Both trials were fully monitored by an external medical monitor.

Blood glucose was measured at routine visits and are random values.

Biopsy-proven acute rejection (BPAR) was graded according to Banff criteria [31].

The primary efficacy endpoint of the ZEUS study was the adjusted eGFR estimated by the Nankivell formula (eGFR, [32]) at month 12. The primary efficacy endpoint in the HERAKLES study was change in eGFR (Nankivell formula) from randomization (month 3) to month 12.

\section{Statistical analysis}

All analyses are reported for the safety populations, comprising all patients who received at least one dose of study drug after study entry. Data on the incidence of PTDM across both studies in patients randomized to everolimus-based CNI-free therapy or standard CsAbased therapy were pooled.

Last observation carried forward (LOCF) method was applied for missing 12-month values for immunosuppression drug doses, drug concentrations, and eGFR. Continuous variables (e.g. drug dose, drug exposure) were compared between groups using the two-sample Wilcoxon rank-sum test of the F-test. The incidence of categorical events in each study and in the pooled analysis was compared between groups using Fisher's test. Kaplan-Meier estimates of time to events were compared between groups using the log rank test. The change in eGFR from randomization to month 12 was analyzed by an ANCOVA model with treatment, center, donor type as factors and eGFR value at randomization as covariate.

All tests were two-sided. $P$ values $<0.05$ were considered significant.

\section{Results}

\section{Patient population and risk factors for diabetes}

In total, 300 patients in the ZEUS study and 497patients in the HERAKLES study were included in the current analysis. Patients were categorized according to whether they developed PTDM or whether they had pre-existing diabetes (Fig. 1).

In both studies, demographic factors, body mass index (BMI), hepatitis C (HCV) status and baseline random blood glucose concentration, generally showed no marked differences between the treatment arms among patients with PTDM, no PTDM or pre-existing diabetes (Table 1). There were significant differences between the 

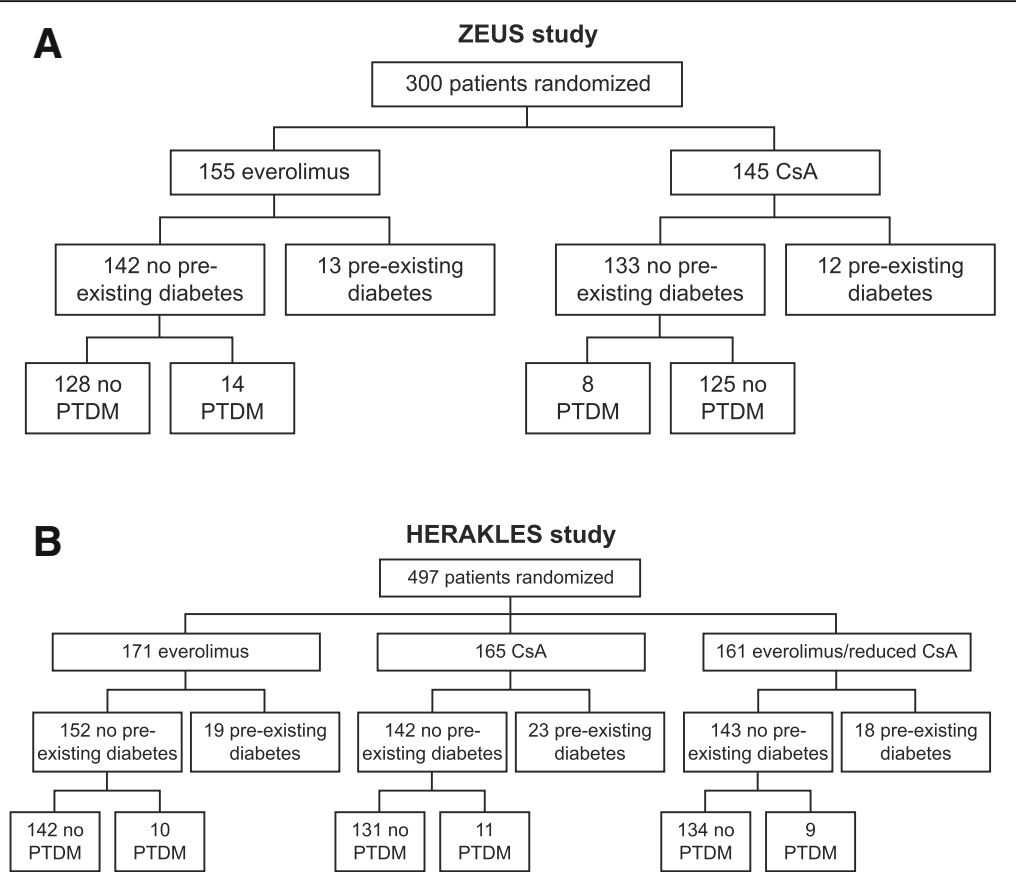

Fig. 1 Patient disposition in (a) the ZEUS study (b) the HERAKLES study (safety populations). PTDM, posttransplant diabetes mellitus

everolimus and CsA cohorts of ZEUS for recipient age among patients with pre-existing diabetes, and for BMI at time of transplant in patients without PTDM and without pre-existing diabetes (Table 1). Almost all patients in both studies were white $(97.5 \%$ [268/275] in ZEUS, 93.6\% [409/437] in HERAKLES).

\section{Immunosuppression}

CsA exposure was comparable between treatment groups at randomization in both trials (Table 2). Oral steroid doses were generally slightly higher after randomization in the everolimus-based CNI-free groups than in CsA-containing regimens within the subpopulations of both trials, with the difference reaching significance in the 'no PTDM' and 'no pre-existing diabetes' groups of ZEUS (Table 2). Use of intravenous steroids to treat rejection before or after randomization was similar between treatment group arms prior to randomization in both trials, and any observed percentage differences within the PTDM and pre-existing diabetes cohorts arose from very small absolute numbers.

\section{Post-transplant diabetes mellitus}

In the ZEUS study, PTDM was present at randomization (i.e. month 4.5 post-transplant) in $9.2 \%$ (13/142) everolimus-treated patients and 5.3\% (7/133) of CsA-treated patients; corresponding values at month 12 were $9.9 \%$ (14/ $142)$ and $6.0 \%(8 / 133)$. In the HERAKLES trial, the incidence of PTDM was 4.6\% (7/152), 6.3\% (9/142) and 4.2\% $(6 / 143)$ at randomization (i.e. month 3 post-transplant) in the everolimus, CsA and everolimus/reduced CsA groups, respectively, compared to $6.6 \%(10 / 152), 7.8 \%(11 / 142)$ and $6.3 \%(9 / 143)$ at month 12 . Thus, after randomization, there were only a total of two new cases of PTDM in the ZEUS study and eight new cases in the HERAKLES trial, distributed equally across treatment groups. Kaplan-Meier estimates showed that there were no significant differences in the incidence of PTDM between treatment groups in either trial (Fig. 2).

When data from both studies were pooled, the incidence of PTDM at month 12 among patients without pre-existing diabetes was $8.2 \%(24 / 294)$ in patients randomized to everolimus without CNI therapy, compared to $6.9 \%(19 / 275)$ in those randomized to standard CsA therapy $(p=0.64)$.

The use of antihyperglycemic therapy was similar between groups for patients with PTDM (everolimus 13/14 [11 insulin, 9 non-insulin therapies] patients, CsA 6/8 [6 insulin, 2 non-insulin therapies] patients) in the ZEUS study, and in the HERAKLES study (everolimus 9/10 [5 insulin, 6 non-insulin therapies], CsA 10/11 [7 insulin, 6 non-insulin therapies], everolimus/reduced CsA 9/9 [8 insulin, 6 non-insulin therapies]).

\section{Pre-existing diabetes}

At time of transplant, pre-existing diabetes was present in $8.4 \%$ and $8.3 \%$ of the everolimus-treated and CsA-treated patients in the ZEUS trial, and in $11.1 \%$, $13.9 \%$ and $11.2 \%$ of the patients randomized to everolimus, CsA or everolimus/reduced CsA in the HERAKLES 


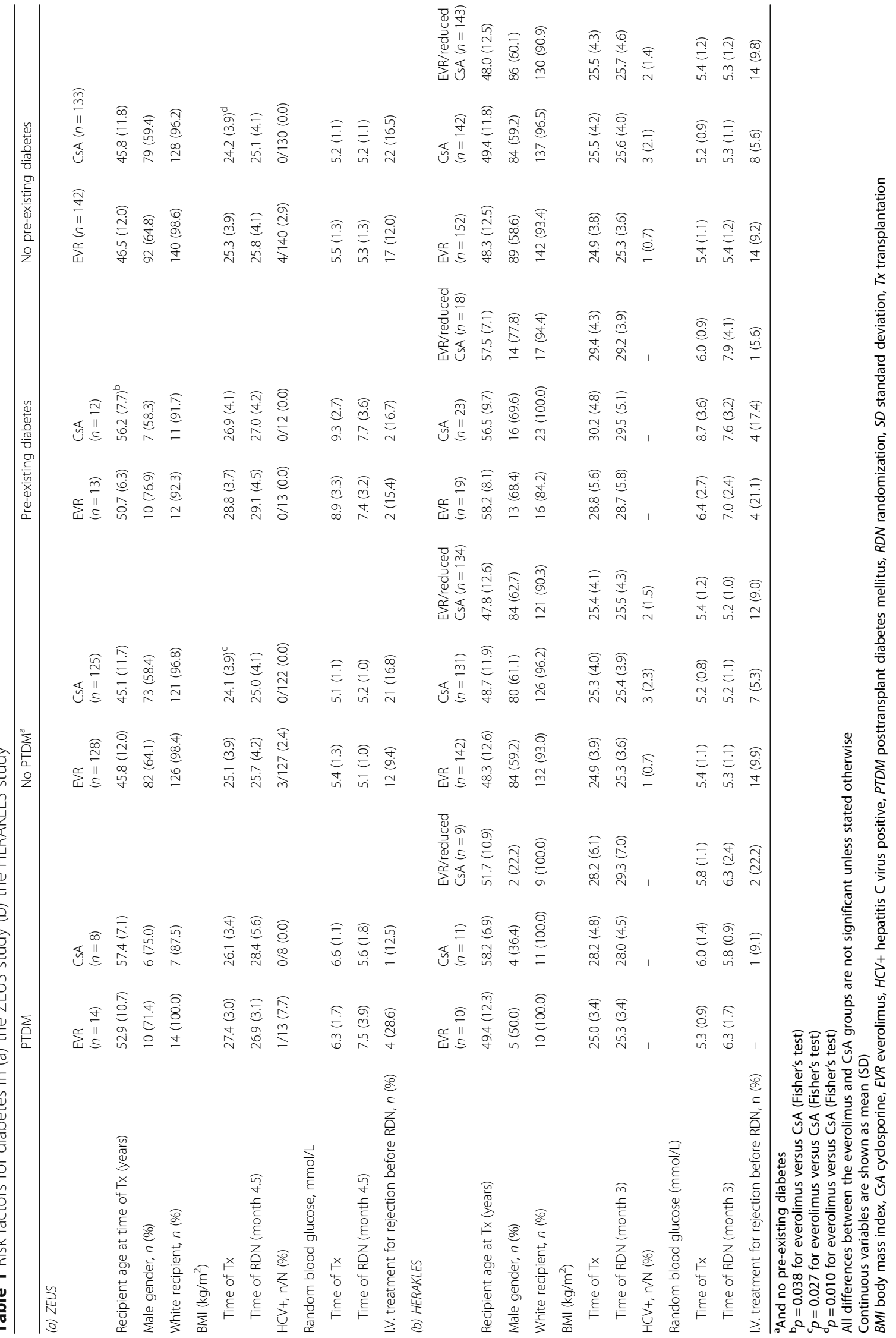




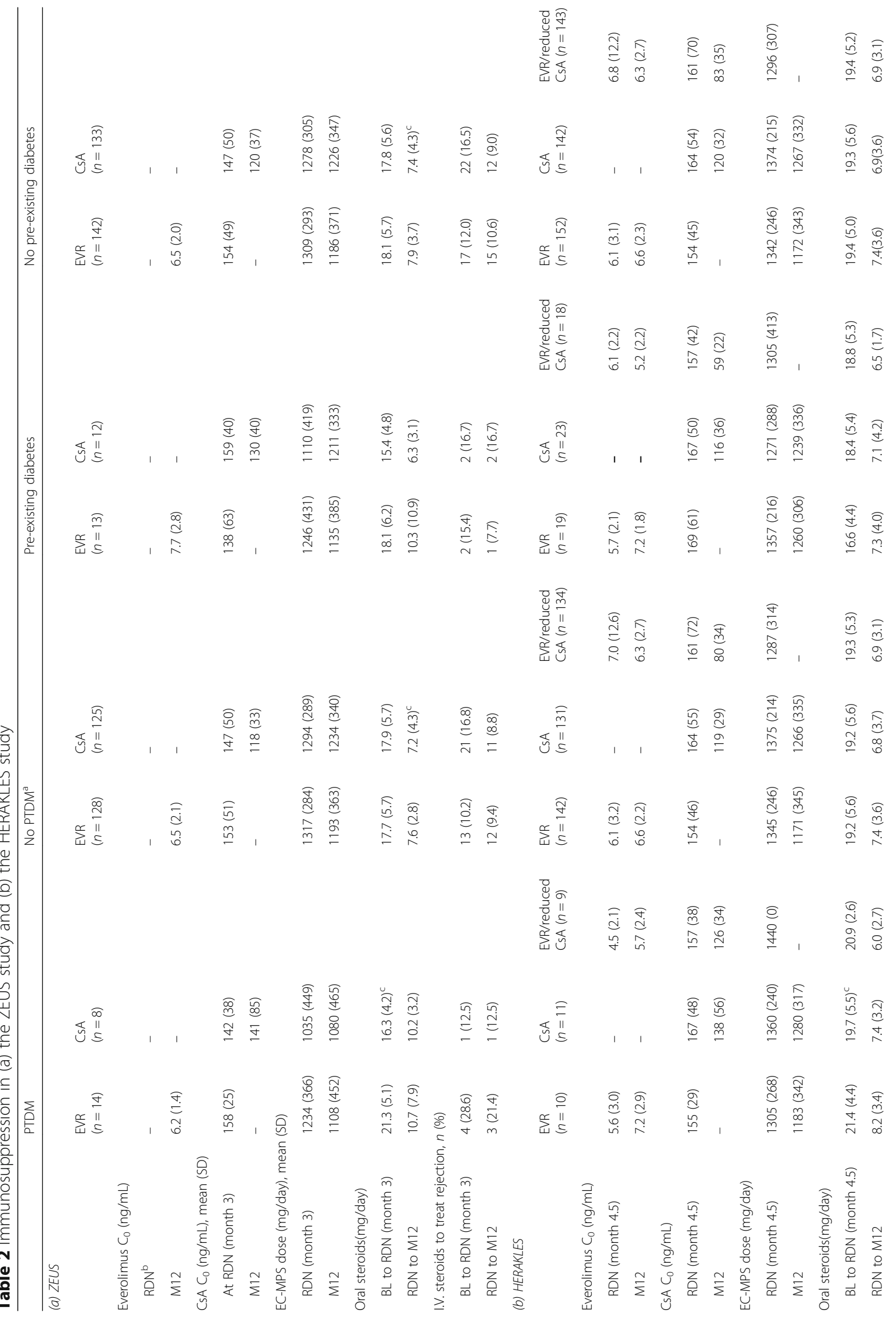




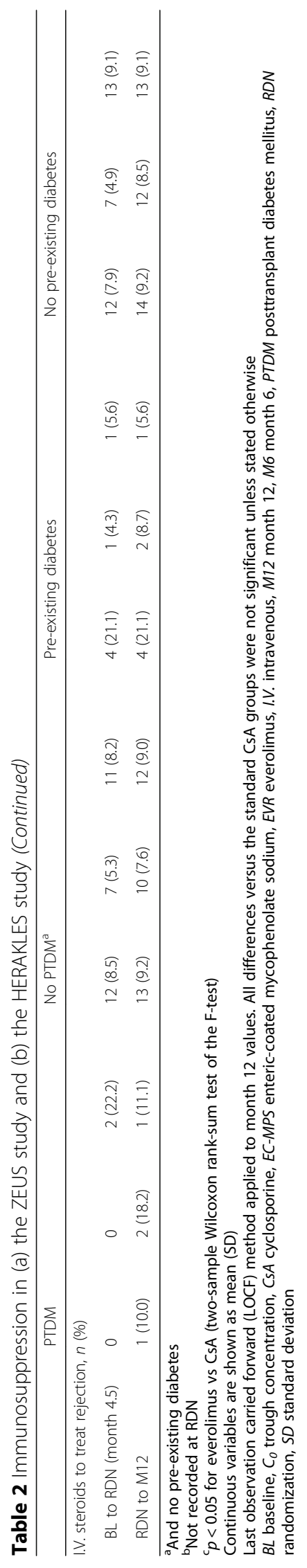




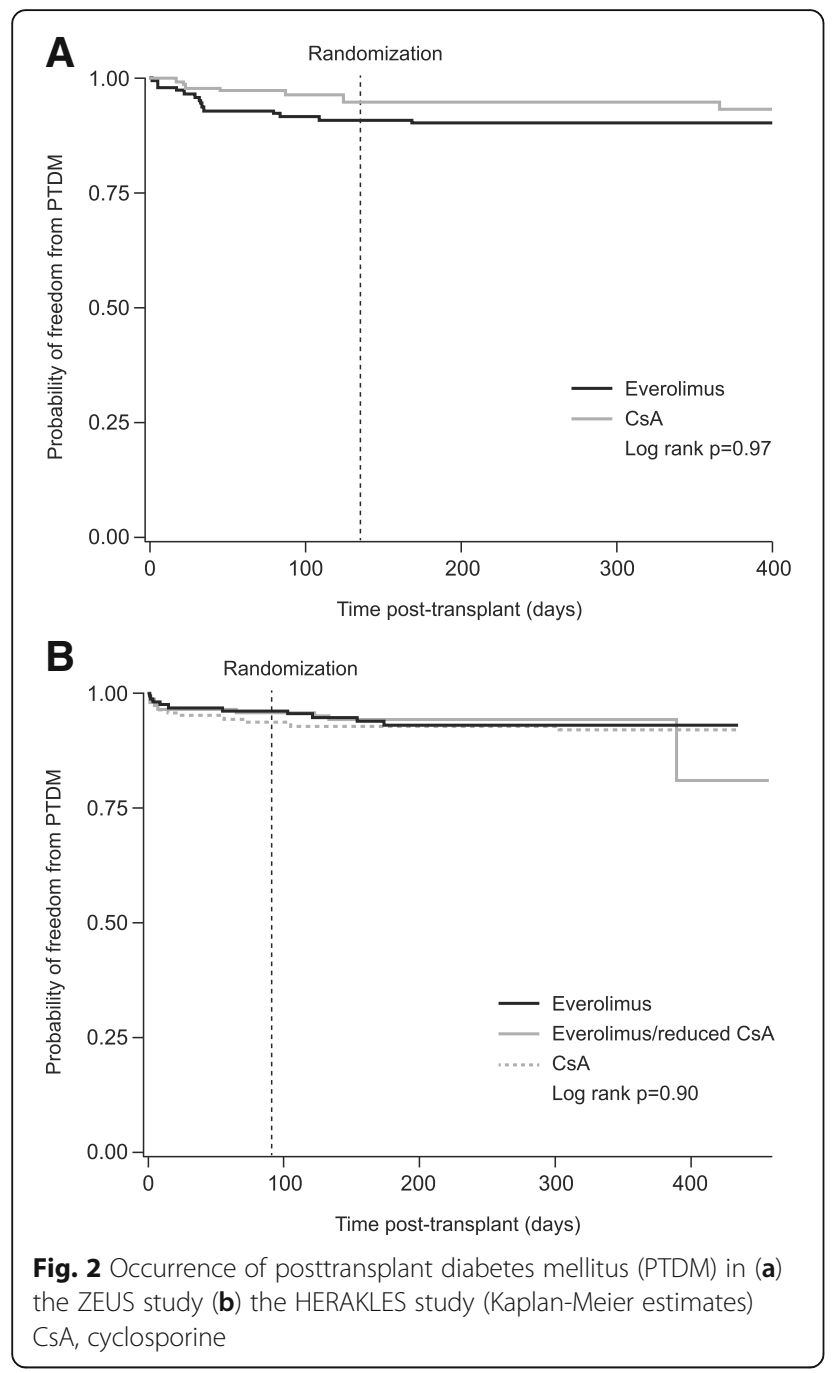

study, respectively. There was no apparent difference in progression of random glucose concentrations between the two treatment groups to month 12 for patients with pre-existing diabetes (Fig. 3). From randomization to month 12 , the mean (SD) change in random glucose concentration was $1.1(3.4) \mathrm{mmol} / \mathrm{L}$ versus $1.5(4.5) \mathrm{mmol} / \mathrm{L}$ in the everolimus versus CsA groups in the ZEUS study ( $p=$ 0.52). In the HERAKLES study, the mean (SD) change was $2.2(3.0) \mathrm{mmol} / \mathrm{L}, 0.5$ (4.9) $\mathrm{mmol} / \mathrm{L}$ and -0.2 (3.7) $\mathrm{mmol} / \mathrm{L}$ in the everolimus, CsA and everolimus/reduced CsA arms, respectively $(p=0.24)$.

Among patients with pre-existing diabetes, use of antihyperglycemic therapy was similar between groups in the ZEUS trial (everolimus 12/13 patients [12 insulin, 3 non-insulin therapies], CsA 12/12 [12 insulin, 4 non-insulin therapies]) and the HERAKLES trial (everolimus 16/19 [16 insulin, 3 non-insulin], CsA 22/23 [20 insulin, 9 non-insulin], everolimus/reduced CsA17/18 [17 insulin, 6 non-insulin]).

\section{Blood glucose concentrations}

The mean (SD) change in random blood glucose from randomization to month 12 among patients with PTDM was similar in the everolimus group versus the CsA group for the ZEUS study $(p=0.10)$ and the HERAKLES study $(p=0.38)$. Mean random blood glucose levels also remained similar between treatment groups in both trials for patients who did not develop PTDM (Fig. 3). As expected, patients with PTDM clearly had higher glucose values compared to patients without PTDM (Fig. 3).

\section{Biopsy-proven acute rejection}

The higher rate of mild BPAR (Grade I) in the overall ZEUS study population was reflected in the cohorts without PTDM or pre-existing diabetes (Additional file 1: Table S2). In the HERAKLES study, there were no significant differences in the incidence of BPAR between treatment groups in any subpopulation (Additional file 1: Table S2).

\section{Renal function}

The change in eGFR from randomization to month 12 was significantly in favor of everolimus in the two largest subpopulations (no PTDM and no pre-existing diabetes) for both trials (Table 3). In the subpopulations with PTDM or pre-existing diabetes, the differences between groups were of a similar order of magnitude to those seen in the larger subpopulations, but in these small cohorts statistical significance was not reached.

\section{Discussion}

Results from this post hoc analysis of two large randomized studies do not suggest any difference in the incidence or severity of PTDM in patients who were converted early post-transplant from a CsA-based regimen to everolimus, or in the progression of pre-existing diabetes. Kaplan-Meier estimates showed comparable rates of PTDM in the different treatment cohorts after randomization in both studies to month 12 , with similar patterns of random blood glucose concentration over time in the subpopulations with or without PTDM or pre-existing diabetes. The number of patients who developed PTDM after randomization was identical in the everolimus group or the standard CsA group, but absolute numbers were very low, even within this large pooled cohort, so firm conclusions cannot be drawn.

The progressive renal deterioration which is frequently associated with diabetes in the general population has also been documented in patients with PTDM [33, 34], so any potential benefit for preservation of renal function may be particularly relevant in this subpopulation. Here, the change in eGFR from randomization to month 12 in patients receiving everolimus within a CNI-free 

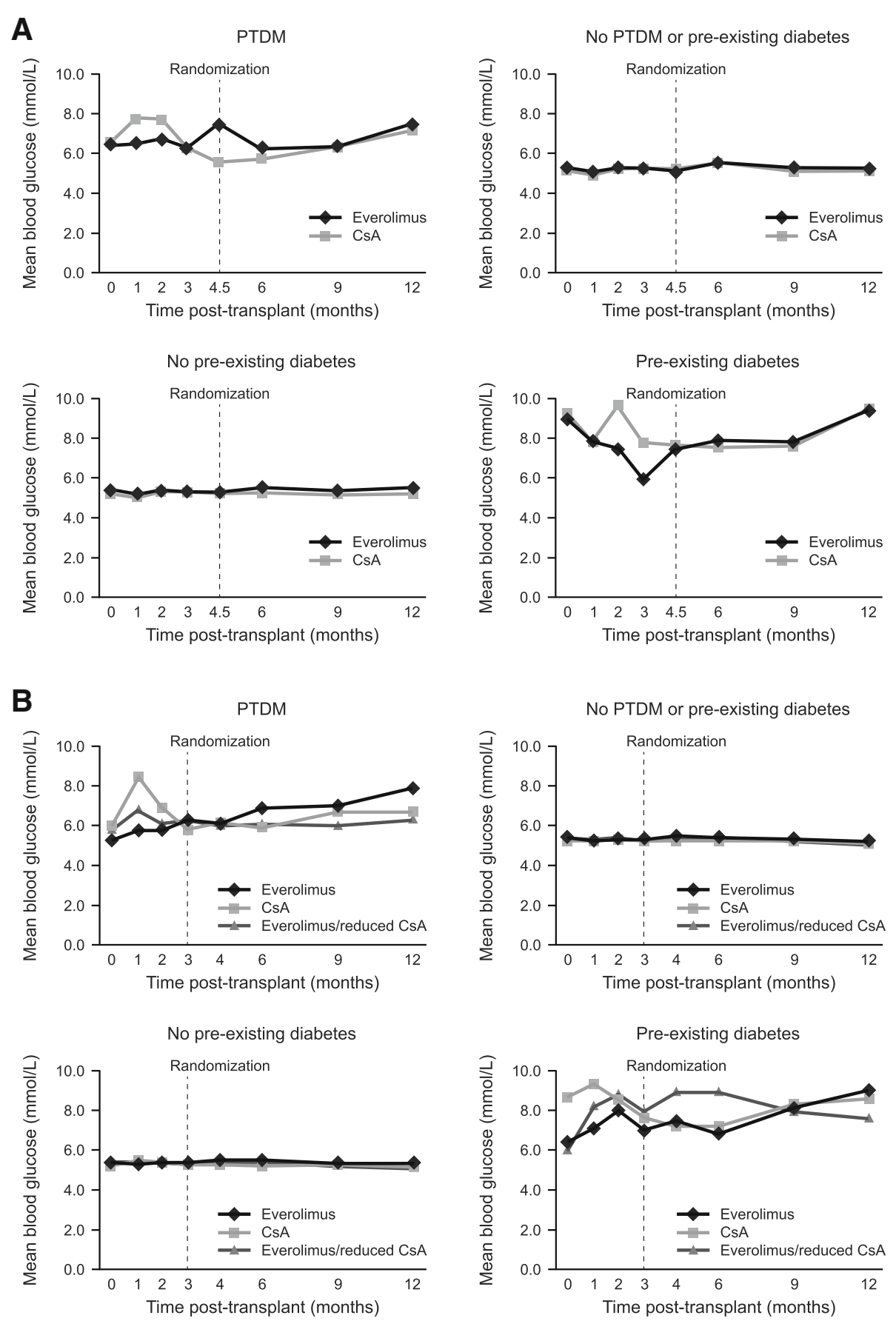

Fig. 3 Mean random blood glucose concentrations from time of transplant to month 12 in (a) the ZEUS study and (b) the HERAKLES study. CSA, cyclosporine; PTDM, posttransplant diabetes mellitus

regimen which was achieved in the overall study populations at month $12[29,30]$ was also observed in the subpopulation of patients with PTDM, although the small numbers of patients precluded any statistical differences. Mean eGFR in patients randomized to CNI-free therapy with everolimus in patients with PTDM improved by $14 \mathrm{~mL} / \mathrm{min} / 1.73 \mathrm{~m}^{2}$ in the ZEUS study and by $4.9 \mathrm{~mL} / \mathrm{min} / 1.73 \mathrm{~m}^{2}$ in HERAKLES by month 12. Among patients with pre-existing diabetes, there was a numerically greater improvement in renal function from randomization to month 12 in the CNI-free cohorts of both trials versus the comparator groups.

Certain aspects of the analysis should be taken into account. First, PTDM was included from the time of 


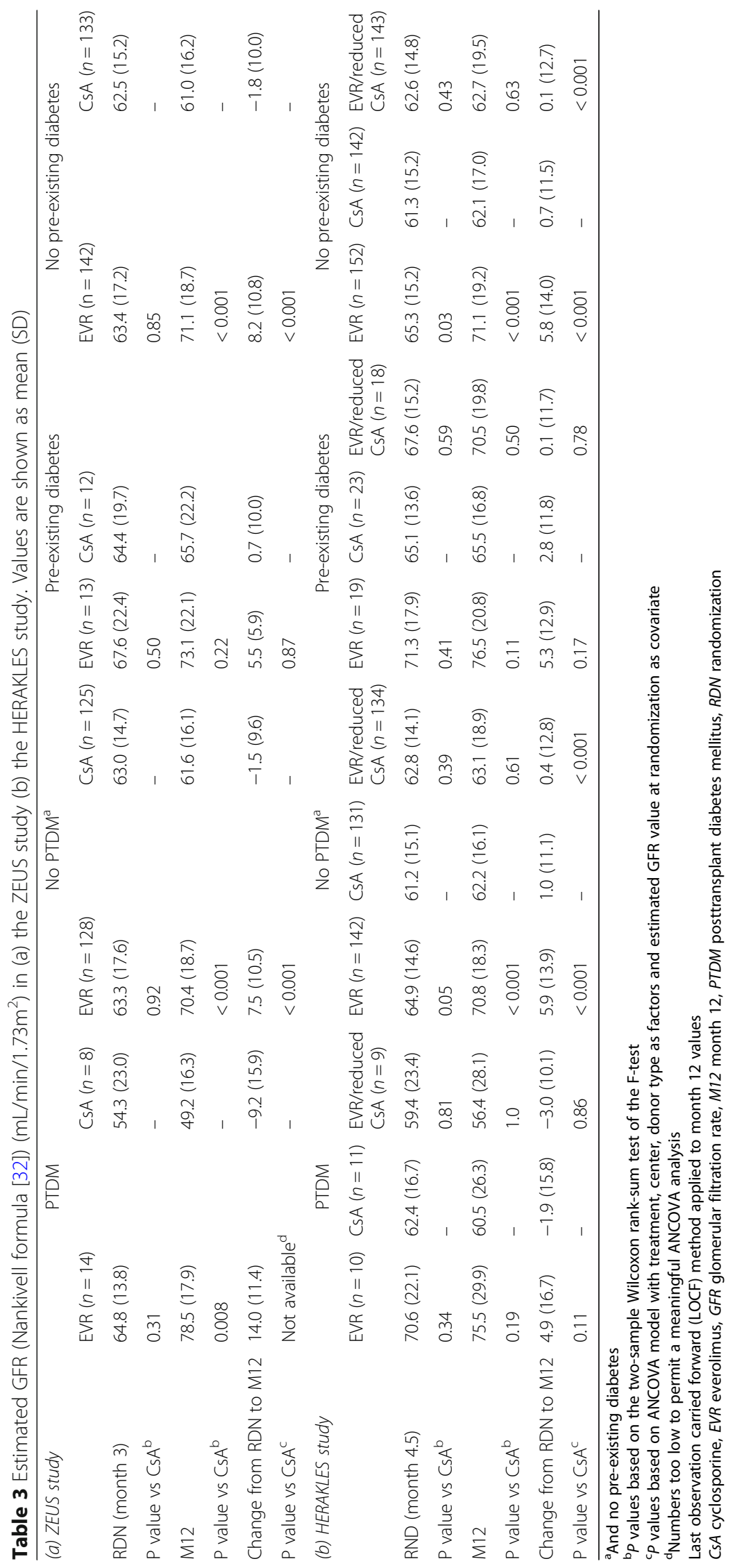


transplant, with few events in either group after randomization, and the onset of PTDM was collected by standard adverse event reporting, with no pre-specified criteria. In the DIRECT study [4], in which data on $\mathrm{HbAl}_{\mathrm{c}}$, insulin level and oral glucose tolerance testing were collected prospectively to month 6 in patients receiving a standard CsA-based regimen, the incidence of treated PTDM was $7.4 \%$ but a further $7.4 \%$ had untreated PTDM based on American Diabetes Association criteria (14.8\% overall) [35]. Based on the 6.8-8.0\% incidence of PTDM reported by 12 months in the current studies, it is likely that the standard reporting adverse event techniques did not capture all cases of PTDM. However, since it seems reasonable to assume that centers applied the same procedures for monitoring and defining PTDM regardless of patients' immunosuppressive treatment, it is unlikely that this will have biased diagnosis rates between treatment arms. Second, oral glucose tolerance tests were not recorded and $\mathrm{HbA}_{\mathrm{c}}$ levels were not measured, with the only available laboratory test being random blood glucose levels. This is a weakness of the analysis. The presence of hyperglycemia could be recorded as an adverse event by investigators, but no strict reporting checks for this value were in place, hence reporting of hyperglycemia is not fully reliable. Information on antidiabetic therapy was captured by standard documentation of concomitant medication, and may have been incomplete despite the strict external monitoring. Third, the analysis does not address the relative diabetogenic effect of everolimus and CsA during the very early post-transplant period (up to month 3 ), when the rate of onset of glucose metabolism disturbances, hyperglycemia and onset of PTDM can be highest $[4,36]$. Fourth, the results presented here apply only to conversion from CsA (not tacrolimus) to everolimus after the initial weeks post-transplant. Tacrolimus, now the dominant $\mathrm{CNI}$, is widely regarded to be more diabetogenic than CsA [37, 38]. Fifth, the studies were not powered to detect differences within subpopulations for the primary endpoint (eGFR at month 12) or to detect differences in any of the endpoints which were specified post hoc. Indeed, even with this large pooled dataset, the number of patients who developed PTDM after randomization was very small, restricting interpretation. Lastly, the follow-up period (a maximum of nine months after randomization) was too short to assess any long-term effect of CNI administration on late-onset PTDM or progression of pre-existing diabetes.

\section{Conclusions}

Within the limitations of this post hoc analysis, including an absence of pre-specified diagnostic criteria for PTDM or extensive laboratory data, conversion from a CsA-based regimen to everolimus in combination with mycophenolic acid and steroids within the first six months after kidney transplantation does not appear to affect the subsequent risk of developing PTDM, or adversely affect the progression of pre-existing diabetes. This finding from the subgroup analysis adds to the observed benefit on renal function after switching to everolimus-based therapy with CNI-withdrawal seen from the main study analyses.

\section{Additional file}

Additional file 1: Table S1. Inclusion and exclusion criteria. Table S2. Efficacy endpoints between randomization and month 12 post-transplant in (a) the ZEUS study (b) the HERAKLES study. (DOCX 24 kb)

\section{Abbreviations}

BMI: Body mass index; BPAR: Biopsy-proven acute rejection; CNI: Calcineurin inhibitor; CSA: Cyclosporine; EC-MPS: Enteric-coated mycophenolate sodium; eGFR: Estimated glomerular filtration rate; HCV: Hepatitis C virus; LOCF: Last observation carried forward; mTOR: Mammalian target of rapamycin; PTDM: Posttransplant diabetes mellitus; SD: Standard deviation

\section{Acknowledgements}

The authors would like to thank Caroline Dunstall for editorial support and Elisabeth Grünewald for statistical support.

\section{Funding}

The ZEUS and HERAKLES studies were funded by Novartis Pharma GmbH, Nürnberg, Germany. The company contributed to the design of the study and to the analysis of data, and reviewed the manuscript before submission. All data collection was undertaken by the investigators.

\section{Availability of data and materials}

The primary results of the two studies reported here are available in reference \#29 (Budde K, Becker T, Arns W, Sommerer C, Reinke P, Eisenberger U. Everolimus-based, calcineurin-inhibitor-free regimen in recipients of de-novo kidney transplants: an open-label, randomised, controlled trial. Lancet 2011;377(9768):837-47) and reference \#30 (Budde K, Zeier M, Witzke O, et al. Everolimus with cyclosporine withdrawal or low-exposure cyclosporine in kidney transplantation from month 3: A multicentre, randomized trial. Nephrol Dial Transplant 2017;32(6):1060-70), and at clinicaltrials.gov (NCT00154310 and NCT00514514) at EudraCT (2006-007021-32 and 2004-004346-40; https:// www.clinicaltrialsregister.eu/ctr-search/trial/2006-007021-32/results. Further information is available on request from Pr C Sommerer (claudia.sommerer@med.uni-heidelberg.de).

\section{Authors' contributions}

CS guided the analysis, developed the PTDM post hoc analysis study design presented here, analyzed the data and wrote the manuscript. CS, OW, FL, WA $P R, U E, B V, K H, J J, M G, R S, I A H, V K, R P W, A M, B S, M D, M Z$, and $K B$ recruited patients and acquired data. CS, E-MP, MP and KB provided medical input, and analyzed the data. CS and MP wrote the manuscript. All authors were involved in drafting the manuscript or revising it critically for important intellectual content. All authors read and approved the final manuscript.

\section{Ethics approval and consent to participate}

All patients provided written informed consent. The study protocols were conducted in compliance with German law and approved by the independent ethics committee or institutional review board for each center, and the procedures followed in the trials were in accordance with the Declaration of Helsinki 1975, as revised in 2008.

Consent for publication

Not applicable.

\section{Competing interests}

Claudia Sommerer has received honoraria from Novartis, Chiesi and Sanofi, and her institution has received research funding from Chiesi, Astellas and Novartis. 
Oliver Witzke has received research funds and/or honoraria from Alexion, Astellas, Bristol-Myers Squibb, Chiesi, Janssen-Cilag, MSD, Novartis, Pfizer, Roche and Shire.

Frank Lehner has received research funds and/or honoraria from Astellas, Bristol-Myers Squibb, Chiesi, Fresenius, Hexal, Novartis, Pfizer and Roche Pharma. Wolfgang Arns has received research funds and/or honoraria from Alexion, Astellas, Chiesi and Novartis.

Petra Reinke has received research funds, honoraria, advisory board from Teva, ThermoFisher, Pfizer, Astellas, Amgen, Baxalta; MSD and Pluriste. Ute Eisenberger has received honoraria and/or travel expenses from Novartis Pharma, Astellas and Amgen.

Bruno Vogt has no conflicts of interest to declare. Katharina Heller has no conflicts of interest to declare. Johannes Jacobi has received honoraria from Roche. Markus Guba has no conflicts of interest to declare Rolf Stahl has no conflicts of interest to declare. Ingeborg A Hauser has received honoraria from Alexion, Astellas, Chiesi, Fresenius, Hexal, Novartis, Roche, Sanofi and Teva. Volker Kliem has received honoraria and fees from Astellas, Novartis, Raptor and Fresenius.

Rudolf $\mathrm{P}$ Wüthrich has received fees for scientific advice from Astellas, Novartis, Roche and Wyeth (now Pfizer).

Anja Mühlfeld has no conflicts of interest to declare. Barbara Suwelack has no conflicts of interest to declare. Michael Duerr has received research funds from Bristol-Myers Squibb and travel grants from Novartis and Roche.

Martin Zeier has received research funding from Dietmar Hopp-Stiftung. Martina Porstner is an employee of Novartis Pharma GmbH.

Eva-Maria Paulus is employee of Novartis Pharma $\mathrm{GmbH}$ and holds stock options.

Klemens Budde has received research funds and/or honoraria from Abbvie, Alexion, Astellas, Bristol-Myers Squibb, Chiesi, Fresenius, Genentech, Hexal, Novartis, Otsuka, Pfizer, Roche, Shire, Siemens, and Veloxis Pharma.

\section{Publisher's Note}

Springer Nature remains neutral with regard to jurisdictional claims in published maps and institutional affiliations.

\section{Author details}

${ }^{1}$ Department of Nephrology, University of Heidelberg, Im Neuenheimer Feld 162, 69120 Heidelberg, Germany. ${ }^{2}$ Department of Infectious Diseases, University Duisburg-Essen, Essen, Germany. ${ }^{3}$ Department of General, Visceral and Transplantation Surgery, Hannover Medical School, Hannover, Germany. ${ }^{4}$ Department of Nephrology and Transplantation, Cologne Merheim Medical Center, Cologne, Germany. ${ }^{5}$ Department of Nephrology and Intensive Care, Charité Campus Virchow, Charité-Universitätsmedizin Berlin, Berlin, Germany. ${ }^{6}$ Department of Nephrology and Hypertension, University of Bern, Inselspital, Bern, Switzerland. ${ }^{7}$ Department of Nephrology and Hypertension, University of Erlangen-Nuremberg, Erlangen, Germany. ${ }^{8}$ Department of General-, Visceral- and Transplantation Surgery, Munich University Hospital, Campus Grosshadern, Munich, Germany. ${ }^{9}$ Division of Nephrology, University Medical Center Hamburg-Eppendorf, Hamburg, Germany. ${ }^{10}$ Med. Klinik III, Department of Nephrology, UKF, Goethe University, Frankfurt, Germany. ${ }^{11}$ Department of Internal Medicine and Nephrology, Kidney Transplant Center, Nephrological Center of Lower Saxony, Klinikum Hann, Münden, Germany. ${ }^{12}$ Division of Nephrology, University Hospital, Zürich, Switzerland. ${ }^{13}$ Division of Nephrology and Immunology, University Hospital RWTH Aachen, Aachen, Germany. ${ }^{14}$ Department of Internal Medicine - Transplant Nephrology, University Hospital of Münster, Münster, Germany. ${ }^{15}$ Department of Nephrology, Charité Universitätsmedizin Berlin, Berlin, Germany. ${ }^{16}$ Novartis Pharma GmbH, Nürnberg, Germany.

\section{Received: 29 August 2017 Accepted: 31 August 2018}

\section{Published online: 19 September 2018}

\section{References}

1. https://optn.transplant.hrsa.gov/data/about-data/optn-database/ (Organ Procurement and Transplantation Network [OPTN] National Data Reports, Waiting list, Organ by diagnosis) Accessed 13 Mar 2016.

2. Adeghate $E$, Schattner $P$, Dunn E. An update on the etiology and epidemiology of diabetes mellitus. Ann N Y Acad Sci. 2006;1084:1-29.
3. Herman $\mathrm{WH}$, Zimmet $\mathrm{P}$. Type 2 diabetes: an epidemic requiring global attention and urgent action. Diabetes Care. 2012;35(5):943-4.

4. Vincenti F, Friman S, Scheuermann E, et al. DIRECT (Diabetes Incidence after Renal Transplantation: Neoral C Monitoring Versus Tacrolimus) Investigators. Results of an international, randomized trial comparing glucose metabolism disorders and outcome with cyclosporine versus tacrolimus. Am J Transplant. 2007;7(6):1506-14.

5. Chadban SJ. New-onset diabetes after transplantation--should it be a factor in choosing an immunosuppressant regimen for kidney transplant recipients. Nephrol Dial Transplant. 2008;23(6):1816-8.

6. Sarno G, Muscogiuri G, De Rosa P. New-onset diabetes after kidney transplantation: prevalence, risk factors, and management. Transplantation. 2012;93(12):1189-95.

7. Boucek P, Saudek F, Pokorna E, et al. Kidney transplantation in type 2 diabetic patients: a comparison with matched non-diabetic subjects. Nephrol Dial Transplant. 2002;17(9):1678-83.

8. Rømming Sørensen V, Schwartz Sørensen S, Feldt-Rasmussen B. Long-term graft and patient survival following renal transplantation in diabetic patients. Scand J Urol Nephrol. 2006;40(3):247-51.

9. Hjelmesaeth J, Hartmann A, Leivestad T, et al. The impact of earlydiagnosed new-onset post-transplantation diabetes mellitus on survival and major cardiac events. Kidney Int. 2006;69(3):588-91.

10. Cosio FG, Kudva Y, van der Velde M, et al. New onset hyperglycemia and diabetes are associated with increased cardiovascular risk after kidney transplantation. Kidney Int. 2005;67(6):2415-21.

11. Burroughs TE, Swindle J, Takemoto $S$, et al. Diabetic complications associated with new-onset diabetes mellitus in renal transplant recipients. Transplantation. 2007;83(8):1027-34.

12. Heisel $O$, Heisel R, Balshaw R, Keown P. New onset diabetes mellitus in patients receiving calcineurin inhibitors: a systematic review and metaanalysis. Am J Transplant. 2004;4(4):583-95.

13. Vesco L, Busson M, Bedrossian J, Bitker MO, Hiesse C, Lang P. Diabetes mellitus after renal transplantation: characteristics, outcome, and risk factors. Transplantation. 1996;61(10):1475-8.

14. Weir MR, Diekmann F, Flechner SM, et al. mTOR inhibition: the learning curve in kidney transplantation. Transpl Int. 2010;23(5):447-60.

15. Sharif A, Shabir S, Chand S, Cockwell P, Ball S, Borrows R. Meta-analysis of calcineurin-inhibitor-sparing regimens in kidney transplantation. J Am Soc Nephrol. 2011;22(11):2107-18.

16. Sharif $A$, Hecking $M$, de Vries AP, et al. Proceedings from an international consensus meeting on posttransplantation diabetes mellitus: recommendations and future directions. Am J Transplant. 2014;14(9):1992-2000.

17. Liefeldt $L$, Budde K. Risk factors for cardiovascular disease in renal transplant recipients and strategies to minimize risk. Transpl Int. 2010;23(12):1191-204.

18. Vitko S, Wlodarczyk Z, Kyllönen L, et al. Tacrolimus combined with two different dosages of sirolimus in kidney transplantation: results of a multicenter study. Am J Transplant. 2006;6(3):531-8.

19. Sampaio EL, Pinheiro-Machado PG, Garcia R, et al. Mycophenolate mofetil vs. sirolimus in kidney transplant recipients receiving tacrolimus-based immunosuppressive regimen. Clin Transpl. 2008;22(2):141-149.

20. Machado PG, Felipe CR, Hanzawa NM, et al. An open-label randomized trial of the safety and efficacy of sirolimus vs. azathioprine in living related renal allograft recipients receiving cyclosporine and prednisone combination. Clin Transpl. 2004;18(1):28-38.

21. Groth CG, Bäckman L, Morales JM, et al. Sirolimus (rapamycin)-based therapy in human renal transplantation: similar efficacy and different toxicity compared with cyclosporine. Sirolimus European Renal Transplant Study group. Transplantation. 1999;67(7):1036-42.

22. Johnston W, Rose CL, Webster AC, Gill JS. Sirolimus is associated with new-onset diabetes in kidney transplant recipients. J Am Soc Nephrol. 2008;19(7):1411-8.

23. Webster AC, Lee WWS, Chapman JR, Craig JC. Target of rapamycin inhibitors (sirolimus and everolimus) for primary immunosuppression of kidney transplant recipients: a systematic review and meta-analysis of randomized trials. Transplantation. 2006;81(9):1234-48.

24. Flechner SM, Glyda M, Cockfield S, et al. The ORION study: comparison of two sirolimus-based regimens versus tacrolimus and mycophenolate mofetil in renal allograft recipients. Am J Transplant. 2011;11(8):1633-44.

25. Ekberg H, Tedesco-Silva H, Demirbas A, et al. ELITE-Symphony Study. Reduced exposure to calcineurin inhibitors in renal transplantation. N Engl J Med. 2007;357(25):2562-75. 
26. Tedesco Silva H Jr, Cibrik D, Johnston T, et al. Everolimus plus reducedexposure CsA versus mycophenolic acid plus standard-exposure CsA in renal-transplant recipients. Am J Transplant. 2010;10(6):1401-13.

27. Qazi Y, Shaffer D, Kaplan B, et al. Efficacy and safety of everolimus plus low-dose tacrolimus versus mycophenolate mofetil plus standard-dose tacrolimus in de novo renal transplant recipients: 12-month data. Am J Transplant. 2017;17(5):1358-69.

28. Rostaing L, Kamar N. mTOR inhibitor/proliferation signal inhibitors: entering or leaving the field? J Nephrol. 2010;23(2):133-42.

29. Budde K, Becker T, Arns W, Sommerer C, Reinke P, Eisenberger U. Everolimus-based, calcineurin-inhibitor-free regimen in recipients of denovo kidney transplants: an open-label, randomised, controlled trial. Lancet. 2011;377(9768):837-47.

30. Budde K, Zeier M, Witzke O, et al. Everolimus with cyclosporine withdrawal or low-exposure cyclosporine in kidney transplantation from month 3: a multicentre, randomized trial. Nephrol Dial Transplant. 2017;32(6):1060-70.

31. Racusen LC, Solez K, Colvin RB, et al. The Banff 97 working classification of renal allograft pathology. Kidney Int. 1999;55(2):713-23.

32. Nankivell BJ, Gruenewald SM, Allen R, Chapman JR. Predicting glomerular filtration rate after kidney transplantation. Transplantation. 1995;59(12):1683-9.

33. Madhav D, Ram R, Dakshinamurty KV. Posttransplant diabetes mellitus: analysis of risk factors, effects on biochemical parameters and graft function 5 years after renal transplantation. Transplant Proc. 2010;42(10):4069-71.

34. Pietrzak-Nowacka M, Safranow K, Dziewanowski K, et al. Impact of posttransplant diabetes mellitus on graft function in autosomal dominant polycystic kidney disease patients after kidney transplantation. Ann Acad Med Stetin. 2008;54(1):41-8.

35. American Diabetes Association. Expert Committee on the Diagnosis and Classification of Diabetes Mellitus.Report of the expert committee on the diagnosis and classification of diabetes mellitus. Diabetes Care. 2003; 26(Suppl 1):S5-20.

36. Luan FL, Stuckey LJ, Ojo AO. Abnormal glucose metabolism and metabolic syndrome in non-diabetic kidney transplant recipients early after transplantation. Transplantation. 2010;89(8):1034-9.

37. Wissing $K M$, Abramowicz D, Weekers $L$, et al. Prospective randomized study of conversion from tacrolimus to cyclosporine A to improve glucose metabolism in patients with posttransplant diabetes mellitus after renal transplantation. Am J Transplant. 2018;18(7):1726-34

38. Knoll GA, Bell RC. Tacrolimus versus cyclosporin for immunosuppression in renal transplantation: meta-analysis of randomised trials. BMJ. 1999; 318(7191):1104-7.

Ready to submit your research? Choose BMC and benefit from:

- fast, convenient online submission

- thorough peer review by experienced researchers in your field

- rapid publication on acceptance

- support for research data, including large and complex data types

- gold Open Access which fosters wider collaboration and increased citations

- maximum visibility for your research: over $100 \mathrm{M}$ website views per year

At BMC, research is always in progress.

Learn more biomedcentral.com/submissions 